\title{
Unusual collateral channel of ruptured vertebral artery fusiform aneurysm involving the posterior inferior cerebellar artery: a case report
}

\author{
Kyung Hoon Kim, Dae Kyun Kim, Suk Jung Jang \\ Department of Neurosurgery, Chosun University College of Medicine, Gwangju, Korea
}

\begin{abstract}
A 60-year-old female patient presented to the emergency department with comatose mentation. Brain computed tomography angiography revealed subarachnoid hemorrhage due to a ruptured right vertebral artery (VA) fusiform aneurysm originating from the posterior inferior cerebellar artery (PICA). Subsequent digital subtraction angiography confirmed that the PICA was supplied by collateral circulation via the ipsilateral posterior meningeal artery. Coil embolization of the ruptured VA fusiform aneurysm was performed. Generally, the PICA is involved in dissecting VA aneurysms, and a therapeutic strategy without reconstructive therapy could pose a significant risk of morbidity. However, this case, we could perform parent artery occlusion without bypass surgery or stenting because of the unusual collateral channel.
\end{abstract}

Keywords: Vertebral artery; Posterior inferior cerebellar artery; Aneurysm

\section{Introduction}

Approximately $10 \%$ of vertebral artery (VA) aneurysms are fusiform and originate from the posterior inferior cerebellar artery (PICA) $[1,2]$. The ideal treatment for fusiform aneurysms is completely isolating them from the circulation while preserving flow to parent vessel branches. Fusiform aneurysms are treated surgically (proximal clip ligation, trapping, and microvascular bypass) or endovascularly (coil embolization, stenting, stent-assisted coiling, and flow diversion) [3-7].

The patient in this study was diagnosed with subarachnoid hemorrhage due to a ruptured right VA fusiform aneurysm originating from the PICA. Digital subtraction angiography (DSA) was performed after confirming subarachnoid hemorrhage using brain computed tomography (CT). We confirmed a preexisting collateral channel between the PICA and posterior meningeal artery

Received: August 11, 2021

Revised: September 6, 2021

Accepted: September 13, 2021

Corresponding Author: Suk Jung Jang, MD, PhD

Department of Neurosurgery, Chosun University College of Medicine, 365

Pilmun-daero, Dong-gu, Gwangju 61453, Korea

Tel: +82-62-220-3126; Fax: +82-62-227-4575;

E-mail: sjajang@chosun.ac.kr
(PMA). Therefore, we performed parent artery occlusion (PAO) without any bypass surgery and stenting because of this unusual collateral channel.

\section{Case Report}

A 60-year-old female patient was admitted to Chosun University Hospital emergency department because of sudden changes in consciousness. The Glasgow Coma Scale score on admission was 3 (E1M1V1).Neurological examination revealed full pupil dilatation and a decerebrate posture. She had no underlying disease or a history of head trauma. Thirty minutes after admission to the emergency department, cardiac arrest occurred. After cardiopulmonary resuscitation, she achieved return of spontaneous circulation but was still hemodynamically unstable.

Brain CT angiography revealed subarachnoid hemorrhage (Fisher grade 4) and right VA fusiform aneurysm incorporated with the PICA (Fig. 1). DSA showed a ruptured VA-PICA aneurysm. This fusiform-shaped aneurysm was likely a dissecting aneurysm, although magnetic resonance imaging was not utilized to confirm an intimal flap and/or intramural hematoma. The PICA was supplied by collateral circulation via the ipsilateral PMA (Fig. 2). 

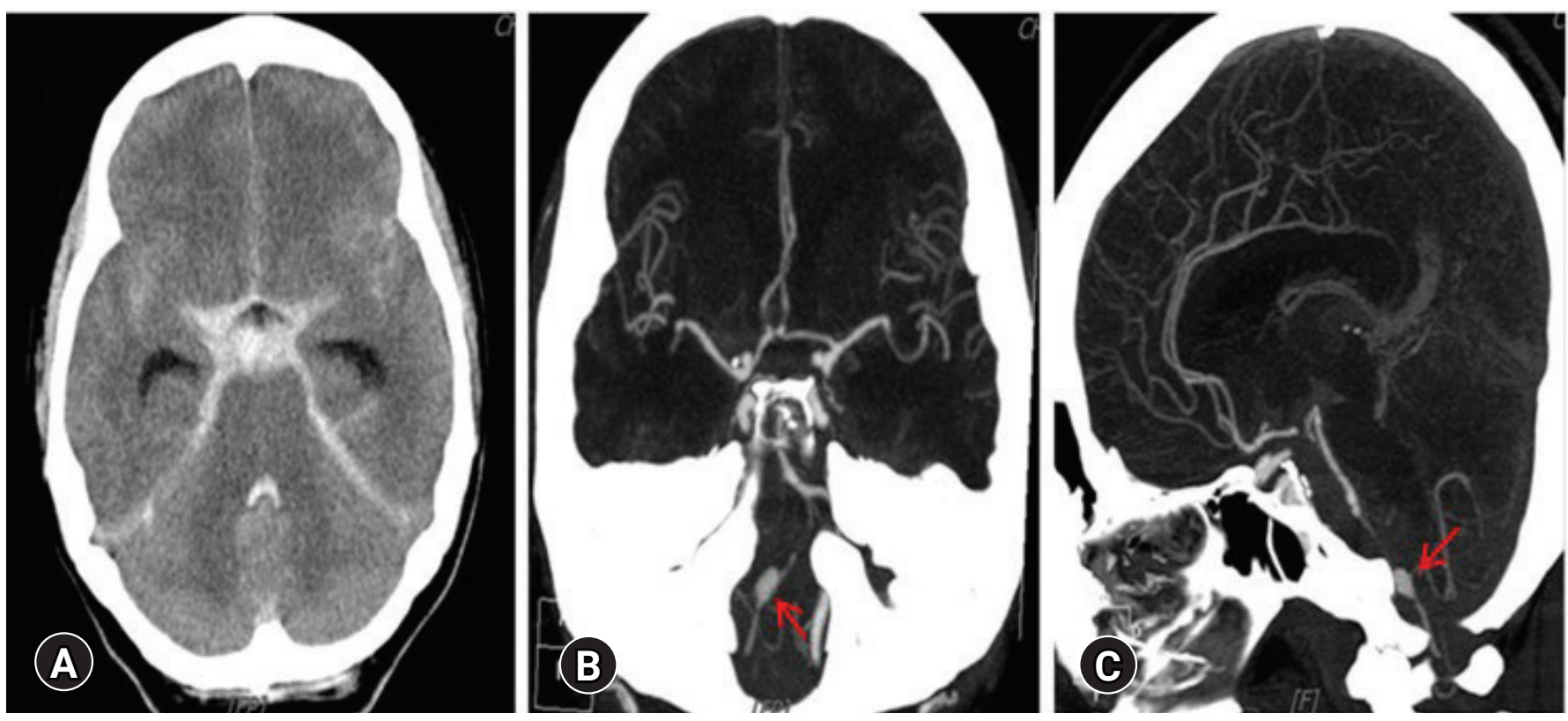

Fig. 1. Brain computed tomography angiography showing (A) a typical subarachnoid hemorrhage with intraventricular hemorrhage and $(B, C)$ a right vertebral artery fusiform aneurysm involving the origin of the posterior inferior cerebellar artery (arrows). Written informed consent was obtained for publication of this case report and accompanying images.
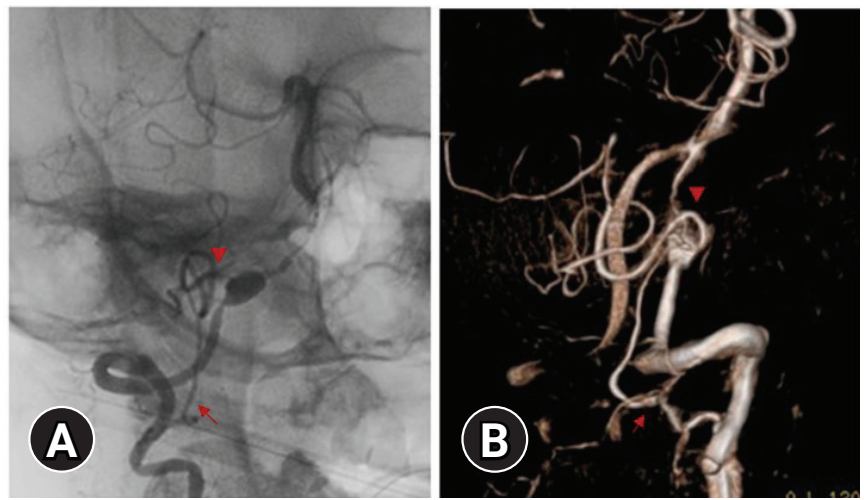

Fig. 2. Digital subtraction angiography showing $(A, B)$ an anastomosis between the posterior inferior cerebellar artery (arrowhead) and the posterior meningeal artery (arrow). Written informed consent was obtained for publication of this case report and accompanying images.

PAO of the right VA and fusiform aneurysm was performed using coils. Follow-up DSA revealed aneurysm occlusion and PICA filling by collateral circulation via the ipsilateral PMA (Fig. 3). An external ventricular drain was performed after the endovascular treatment.

The patient recovered slowly, and she was able to return home with no focal neurological deficits after one month of rehabilitation. She has been capable of performing daily living activities since. One year postoperative, brain magnetic resonance imaging showed no interval change.
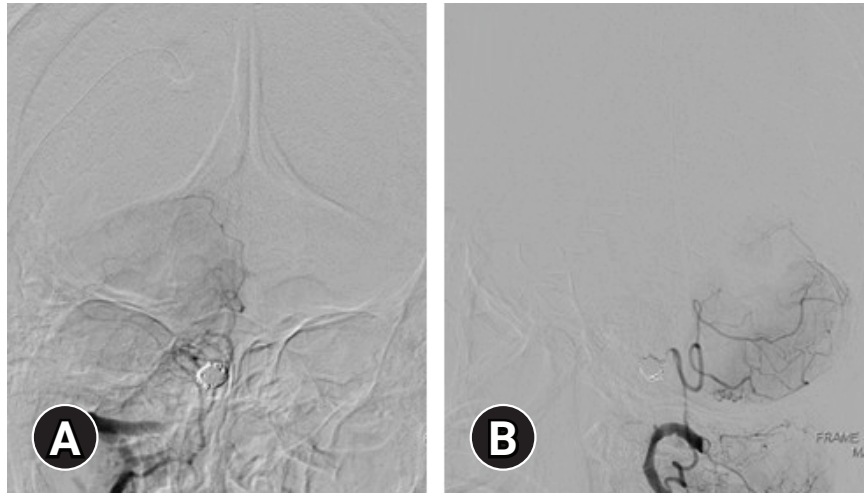

Fig. 3. Postoperative digital subtraction angiography showing (A) parent vessel occlusion of the right vertebral artery and fusiform aneurysm and (B) filling of the posterior inferior cerebellar artery territory by collateral circulation via the ipsilateral posterior meningeal artery. Written informed consent was obtained for publication of this case report and accompanying images.

\section{Discussion}

Intracranial aneurysms can be classified according to their pathogenesis, shape, or etiology. Classification according to its form is the most used, and it can be divided into saccular and nonsaccular types. Fusiform aneurysms are nonsaccular and involve the vessel wall at a variable distance and can present different formation processes. The two principal causes of this type of aneurysm are dissection and atherosclerosis; disorders of collagen and elastin 
metabolism, by infections, very rarely by neoplastic invasion of the arterial wall and iatrogenesis are other origins of this vasculopathy [8]. Fusiform aneurysms represent about 3\% to $13 \%$ of all intracranial aneurysms, and they are often observed at the vertebrobasilar system $[8,9]$. Approximately $10 \%$ of VA aneurysms are fusiform and originate from the PICA [1,2]. Fusiform aneurysms have different underlying pathologies, hemodynamics, anatomical distributions, natural histories, and treatments compared to the saccular variety [8].The ideal treatment for fusiform aneurysms is completely isolating them from the circulation while preserving flow to parent vessel branches. Surgical treatments, proximal clip ligation, trapping, and microvascular bypass have classically been the method of choice for fusiform aneurysms. Some of these strategies achieve exclusion of the aneurysm from the circulation, while others depend on flow reversal to decrease the risk of future rupture. Endovascular treatments, including parent vessel coil embolization, stenting, stent-assisted coiling, and flow diversion, have recently emerged as alternatives to open surgery [3-7]. The patient in this study was hemodynamically unstable and therefore required urgent evaluation and treatment. DSA showed a VA fusiform aneurysm originating from the PICA, which was supplied by collateral circulation via the ipsilateral PMA. PVO of the right VA and fusiform aneurysm was performed using coils.

PMA arose from the third segment of the VA. After entering the skull, the PMA courses within the dura mater and supplies the occipital dura mater, falx cerebelli, tentorium cerebelli, and falx cerebri. Normally, there is no anastomosis between the PICA and PMA. However, four cases of PMA originating from the PICA have been reported $[10,11]$ and one case of PICA originating from the PMA [12]. In the $12-\mathrm{mm}$ to $20-\mathrm{mm}$ embryo stages, the primitive cranial vessels differentiate into the external, dural, and cerebral vessels. Anastomoses exist among these three vascular systems prior to differentiation. Some anastomoses may present between the primitive vessels forming the PICA and the PMA and sometimes persist until adulthood [11]. However, the delayed development of collateral circulation from the PMA to the PICA has not been reported. We confirmed the presence of PMA through DSA. If the PICA is clogged, it can be life-threatening. However, in our case, effective embolization was possible. Despite being uncommon, PMA should be considered as a potential collateral channel to the PICA in therapeutic proximal PICA occlusion.

\section{Conclusion}

Generally, there is no anastomosis between the PICA and PMA.
In a case like this, the PAO for a ruptured VA dissecting aneurysm involving the origin of the PICA is effective.

\section{Conflicts of interest}

No potential conflict of interest relevant to this article was reported.

\section{Acknowledgments}

This study was supported by the Research Fund of Chosun University Hospital, 2021.

\section{ORCID}

Kyung Hoon Kim, https://orcid.org/0000-0002-6913-060X

Dae Kyun Kim, https://orcid.org/0000-0002-3636-002X

Suk Jung Jang, https://orcid.org/0000-0003-4767-1330

\section{REFERENCES}

1. George B, Bruneau M, Spetzler RF. Pathology and surgery around the vertebral artery. Paris: Springer-Verlag France; 2011.

2. Lehto H, Niemelä M, Kivisaari R, et al. Intracranial vertebral artery aneurysms: clinical features and outcome of 190 patients. World Neurosurg 2015;84:380-9.

3. Aihara M, Naito I, Shimizu T, et al. Predictive factors of medullary infarction after endovascular internal trapping using coils for vertebral artery dissecting aneurysms. J Neurosurg 2018;129: $107-13$.

4. Awad AJ, Mascitelli JR, Haroun RR, De Leacy RA, Fifi JT, Mocco J. Endovascular management of fusiform aneurysms in the posterior circulation: the era of flow diversion. Neurosurg Focus 2017;42:E14.

5. Dabus G, Lin E, Linfante I. Endovascular treatment of fusiform intracranial vertebral artery aneurysms using reconstructive techniques. J Neurointerv Surg 2014;6:589-94.

6. Li W, Liu J, Zhang Y, et al. Flow diversion and outcomes of vertebral fusiform aneurysms after stent-only treatment: a hemodynamic study. World Neurosurg 2017;107:202-10.

7. Satow T, Ishii D, Iihara K, Sakai N; JR-NET Study Group. Endovascular treatment for ruptured vertebral artery dissecting aneurysms: results from Japanese Registry of Neuroendovascular Therapy (JR-NET) 1 and 2. Neurol Med Chir (Tokyo) 2014; 54:98-106.

8. Park SH, Yim MB, Lee CY, Kim E, Son EI. Intracranial fusiform aneurysms: it's pathogenesis, clinical characteristics and manage- 
ments. J Korean Neurosurg Soc 2008;44:116-23.

9. Onofrj V, Cortes M, Tampieri D. The insidious appearance of the dissecting aneurysm: Imaging findings and related pathophysiology: a report of two cases. Interv Neuroradiol 2016;22: 638-42.

10. Okuno S, Touho H, Ohnishi H, Karasawa J. Ruptured aneurysm at the bifurcation of the posterior meningeal artery from the proximal posterior inferior cerebellar artery. Acta Neurochir (Wien) 1998;140:629-30.
11. Tanohata K, Maehara T, Noda M, Katoh H, Sugiyama S, Okazaki A. Anomalous origin of the posterior meningeal artery from the lateral medullary segment of the posterior inferior cerebellar artery. Neuroradiology 1987;29:89-92.

12. Ogawa T, Fujita H, Inugami A, Shishido F, Higano S, Uemura K. Anomalous origin of the posterior inferior cerebellar artery from the posterior meningeal artery. AJNR Am J Neuroradiol 1991; 12:186. 